\title{
B-type natriuretic peptide levels predict outcomes in infants undergoing cardiac surgery in a lesion-dependent fashion
}

\author{
Rambod Amirnovin, MD, ${ }^{\text {a,b }}$ Roberta L. Keller, MD, ${ }^{a}$ Christina Herrera, BA, ${ }^{\mathrm{b}}$ Jong-Hau Hsu, MD, ${ }^{\mathrm{c}}$ \\ Sanjeev Datar, MD, PhD, ${ }^{\mathrm{a}}$ Tom R. Karl, MD, ${ }^{\mathrm{d}}$ Ian Adatia, MBChB, ${ }^{\mathrm{e}}$ Peter Oishi, MD, ${ }^{\mathrm{a}, \mathrm{b}}$ and \\ Jeffrey R. Fineman, MD ${ }^{\text {a,b }}$
}

\begin{abstract}
Objective: B-type natriuretic peptide is used in the diagnosis, risk stratification, and management of adult patients with cardiac disease. However, its use in infants with congenital heart disease has been limited, particularly in the perioperative period. Our objective was to determine the alterations in perioperative B-type natriuretic peptide levels and their predictive value on postoperative outcomes, in infants undergoing congenital heart surgery.
\end{abstract}

Methods: We prospectively enrolled 115 patients: 24 with univentricular heart disease undergoing a modified Norwood procedure, 11 with d-transposition of the great arteries, 55 with hemodynamically important left-toright shunt, and 25 with tetralogy of Fallot undergoing primary repair. Clinical data and B-type natriuretic peptide samples were collected before and 2, 12, and 24 hours after cardiopulmonary bypass. Univariate analysis and multivariate linear regression analysis were performed.

Results: The perioperative B-type natriuretic peptide levels were lesion specific. Patients with d-transposition of the great arteries and univentricular heart disease had high preoperative B-type natriuretic peptide levels that decreased postoperatively, and those with hemodynamically important left-to-right shunts and tetralogy of Fallot had lower preoperative levels that increased during the first 12 hours postoperatively. The patients with univentricular heart disease with an adverse outcome had a significantly greater 24-hour B-type natriuretic peptide level than those without $(P<.05)$. Those with hemodynamically important left to right shunts and an adverse outcome had a greater 12-hour B-type natriuretic peptide level than those without $(P<.05)$. A 12-hour postoperative/ preoperative ratio greater than 45 was $100 \%$ sensitive and $82 \%$ specific for an adverse outcome in the patients with tetralogy of Fallot.

Conclusions: The perioperative changes in B-type natriuretic peptide levels and their ability to predict outcomes are lesion-specific. Characterization of these changes might be useful in caring for infants after congenital heart surgery. (J Thorac Cardiovasc Surg 2013;145:1279-87)

Surgical repair or palliation of congenital cardiac defects is performed routinely in the first year of life. However, the perioperative management remains challenging, and

\footnotetext{
From the Department of Pediatrics ${ }^{\mathrm{a}}$ and Cardiovascular Research Institute, ${ }^{\mathrm{b}}$ University of California, San Francisco, Calif; Department of Pediatrics, ${ }^{c}$ Kaohsiung Medical University Hospital, Kaohsiung, Taiwan; Queensland Pediatric Cardiac Services Mater Children's Hospital, ${ }^{\mathrm{d}}$ South Brisbane, Queensland, Australia; and Department of Pediatrics, ${ }^{\mathrm{e}}$ University of Alberta, Edmonton, Alberta, Canada

This research was supported in part by University of California, San FranciscoClinical \& Translational Science Institute grant UL1 RR024131 from the National Institutes of Health and National Center for Research Resources, grants KO8 HD047349 (to P.O.), K23 HL079922 (to R.L.K.), and HL61284 (to J.R.F.) from the National Institutes of Health, and a grant from the Foundation Leducq (to J.R.F.) and the Pulmonary Hypertension Association (to R.L.K.). J.-H. Hsau was supported in part by the Department of Pediatrics, Kaohsiung Medical University Hospital, Kaohsiung, Taiwan.

Disclosures: Authors have nothing to disclose with regard to commercial support.

Received for publication Dec 23, 2011; revisions received June 25, 2012; accepted for publication July 26, 2012; available ahead of print Aug 22, 2012.

Address for reprints: Jeffrey R. Fineman, MD, Department of Pediatrics, University of California, San Francisco, School of Medicine, 513 Parnassus Avenue, Box 1346, San Francisco, CA 94143-1346 (E-mail: jeff.fineman@ucsf.edu). $0022-5223 / \$ 36.00$

Copyright (c) 2013 by The American Association for Thoracic Surgery http://dx.doi.org/10.1016/j.jtcvs.2012.07.067
}

outcomes are often unpredictable. Neonates and infants experience adverse events more often and have greater perioperative mortality than older children. Although riskadjustment scoring systems and various biomarkers have been investigated, it remains difficult to identify individual patients with an increased risk of a poor postoperative outcome. $^{1,2}$

B-type natriuretic peptide (BNP) is commonly used as a biomarker for the diagnosis, risk stratification, and management of adult heart failure, but a similar use in neonates and infants undergoing surgical repair or palliation of congenital cardiac defects has not yet been established. ${ }^{3-7}$ However, several recent studies of this patient population have found that perioperative BNP levels were predictive of important clinical outcomes. These studies also suggested that age and the specific cardiac defect type might significantly influence the manner in which BNP levels related to clinical outcomes. Thus, it appears that the assay of BNP as a part of perioperative management offers potential benefit. However, additional study is necessary to determine how best to use the BNP determinations across the spectrum of patients undergoing 


$$
\begin{aligned}
& \text { Abbreviations and Acronyms } \\
& \begin{aligned}
\mathrm{AVdO}_{2}= & \text { arterial-venous oxygen saturation } \\
& \text { difference } \\
\mathrm{BNP} & =\text { B-type natriuretic peptide } \\
\mathrm{CPB} & =\text { cardiopulmonary bypass } \\
\mathrm{LCOS} & =\text { low cardiac output syndrome } \\
\mathrm{LRS} & =\text { left to right shunt } \\
\mathrm{TGA} & =\text { d-transposition of the great arteries } \\
\mathrm{TOF} & =\text { tetralogy of Fallot } \\
\mathrm{UVH} & =\text { univentricular heart }
\end{aligned}
\end{aligned}
$$

surgery for the repair or palliation of congenital cardiac defects. ${ }^{8,9}$

Therefore, the objectives of the present study were to determine and compare the perioperative BNP levels and the potential predictive value of these levels for the postoperative outcome in 2 groups of neonates and 2 groups of infants, including 4 cardiac defects with distinct perioperative physiologic features. We hypothesized that BNP levels would be associated with clinical outcomes in an age- and cardiac defect-dependent manner.

\section{METHODS}

We conducted a prospective cohort study in the pediatric cardiac intensive care unit at the University of California, San Francisco, Benioff Children's Hospital between June 2005 and October 2009. The eligible patients were neonates and infants with congenital cardiac defects undergoing surgical repair or palliation using cardiopulmonary bypass (CPB). To eliminate some potentially confounding variables, patients were excluded from the study if they weighed less than $2 \mathrm{~kg}$, were older than 12 months, or were less than 36 weeks postmenstrual age at surgery.

We prospectively studied 115 neonates and infants. To compare single ventricle and biventricular disease, neonates with univentricular heart disease (UVH), undergoing a modified Norwood procedure, were studied and compared with neonates with d-transposition of the great arteries (TGA), undergoing an arterial switch operation. Similarly, to assess the differences related to preoperative pulmonary blood flow, infants with large left-toright shunts (LRSs), undergoing primary repair, were studied and compared with infants with tetralogy of Fallot (TOF), undergoing primary repair. In all 4 groups, systemic arterial plasma BNP determinations were made before and 2, 12, and 24 hours after CPB.

The preoperative anesthesia management, intraoperative bypass strategy, and subsequent pediatric cardiac intensive care unit management followed standard institutional practices. The clinical team, which was unaware of the BNP values, made all patient management decisions. The study was conducted under approval of the University of California, San Francisco, institutional review board. The patients' parents or guardians provided written informed consent before enrollment.

\section{Patient Classification}

We divided the patients into 4 groups according to the preoperative echocardiographic findings and intraoperative verification by the surgeon: group I (UVH) included neonates with single ventricle physiology undergoing palliation with a modified Norwood procedure, group II (TGA) included neonates undergoing an arterial switch procedure for TGA; group
III (LRS) included those with LRSs, including large ventricular septal defects and balanced atrioventricular septal defects undergoing biventricular repair; and group IV (TOF) included infants undergoing biventricular repair of TOF.

\section{Data Collection}

Blood samples were obtained from an arterial catheter preoperatively (before skin incision) and at 2,12, and 24 hours after CPB. The BNP levels were measured using a commercially available fluorescence immunoassay (Triage Meter Plus; Biosite Diagnostic, San Diego, Calif). The measurable range of $\mathrm{BNP}$ on this device is 5 to $5000 \mathrm{pg} / \mathrm{mL}$. The coefficient of variation for the assay is $9.2 \%$ to $11.4 \%$.

Clinical and biochemical data were collected prospectively at each sampling point and daily thereafter. The clinical data collected included patient demographics, CPB duration, aortic crossclamp duration, inotrope dose, mean systemic arterial pressure, central venous pressure, heart rate, arterial saturation using pulse oximetry, cerebral oxygen saturation using near infrared spectroscopy, intensive care unit duration, hospital stay, and mechanical ventilation duration. The biochemical data collected included hematocrit, arterial and venous blood gases, serum lactate, blood urea nitrogen, and creatinine.

\section{Outcome Measures}

The primary endpoint was the occurrence of a major poor postoperative outcome within 6 months of surgery, defined as death, the need for an unplanned invasive catheter-based or surgical intervention, the need for extracorporeal life support (ECLS) after surgery, or the need for cardiac transplantation.

The secondary endpoints included the mechanical ventilation duration, duration of hospitalization, and development of low cardiac output syndrome (LCOS) within 48 hours after surgery. The definition of LCOS was derived from criteria published by Hoffman and colleagues, ${ }^{10}$ that included a combination of changes in clinical signs and laboratory indicators. The criteria included tachycardia, oliguria, poor perfusion, cardiac arrest, or metabolic acidosis and the need for interventions aimed at augmenting cardiac output, such as increased pharmacologic support relative to baseline and cardiac pacing.

\section{Calculations}

Inotrope use was quantified by a score adapted from Wernovsky and colleagues. ${ }^{11}$ The score was calculated from the level of inotropic support the patients were receiving (in $\mu \mathrm{g} / \mathrm{kg} / \mathrm{min}$ ), at each sampling point according to the following equation: dopamine + dobutamine + [(epinephrine + norepinephrine $) \times 100]+($ milrinone $\times 20)$. The arterial-venous oxygen saturation difference $\left(\mathrm{AVdO}_{2}\right)$ was calculated as the co-oximetric arterial oxyhemoglobin saturation minus the central venous oxyhemoglobin saturation.

\section{Statistical Analysis}

Differences in the continuous variables between groups were tested with the Student $t$ test for normally distributed variables and the MannWhitney $U$ test for non-normally distributed variables. Differences in the categorical variables between groups were tested with Fisher's exact test. Contingency tables were formulated to determine the sensitivity and specificity of the BNP measurements. Correlations between variables were performed using the Spearman rank correlation method. The predictive value of BNP for the primary and secondary outcomes was tested for potential demographic and physiologic confounders with multivariate regression analysis when adequate outcomes were available. Changes in BNP over time were compared by repeated measures analysis of variance. Statistical analyses were performed with the use of Prism, version 4.0 (GraphPad Software, Inc, San Diego, Calif) and Stata, version 11.0 (StataCorp, College Station, Tex). 
TABLE 1. Preoperative demographics

\begin{tabular}{|c|c|c|c|c|c|c|}
\hline \multirow[b]{2}{*}{ Variable } & \multicolumn{3}{|c|}{ Neonates } & \multicolumn{3}{|c|}{ Infants } \\
\hline & $\begin{array}{c}\text { Group I } \\
\text { (UVH) }\end{array}$ & $\begin{array}{c}\text { Group II } \\
\text { (TGA) }\end{array}$ & $P$ value* & $\begin{array}{c}\text { Group III } \\
\text { (LRS) }\end{array}$ & $\begin{array}{c}\text { Group IV } \\
\text { (TOF) }\end{array}$ & $P$ value* \\
\hline Patients & 24 & 11 & & 55 & 25 & \\
\hline Age & $9 \pm 8 d$ & $10 \pm 9 \mathrm{~d}$ & .87 & $10 \pm 2 \mathrm{mo}$ & $4 \pm 3 \mathrm{mo}$ & .02 \\
\hline Weight $(\mathrm{kg})$ & $3.3 \pm 0.4$ & $3.5 \pm 0.4$ & .26 & $6.4 \pm 1.4$ & $5.2 \pm 1.5$ & .09 \\
\hline Males (n) & $10(42 \%)$ & $10(91 \%)$ & $<.01$ & $28(50 \%)$ & $16(64 \%)$ & .28 \\
\hline Surgery type & Norwood $\dagger$ & Arterial switch & & Primary repair & Primary repair & \\
\hline Cardiac lesion & $\begin{array}{c}15 \text { HLHS; } 3 \text { Shone's complex; } \\
2 \text { IAA, HAA; } 2 \text { DORV, HRV, } \\
\text { HAA; } 2 \text { tricuspid atresia, } \\
\text { TGA, HRV, HAA }\end{array}$ & 11 d-TGA & & 40 VSD 15 AVSD & $25 \mathrm{TOF}$ & \\
\hline \multicolumn{7}{|l|}{ Preoperative data } \\
\hline $\operatorname{HR}\left(\min ^{-1}\right)$ & $151 \pm 16$ & $147 \pm 25$ & .6 & $134 \pm 20$ & $135 \pm 18$ & .8 \\
\hline MAP (mm Hg) & $49 \pm 7$ & $48 \pm 5$ & .6 & $62 \pm 12$ & $61 \pm 15$ & .7 \\
\hline $\begin{array}{l}\text { Pulse oximetry } \\
\qquad(\% \text { saturation })\end{array}$ & $91 \pm 6$ & $89 \pm 6$ & .3 & $98 \pm 0.4$ & $94 \pm 1$ & $<.01$ \\
\hline Mechanical ventilation & $16(67 \%)$ & $10(91 \%)$ & .4 & $4(7 \%)$ & $4(30 \%)$ & .4 \\
\hline $\mathrm{PGE}_{1}$ & $21(88 \%)$ & $11(100 \%)$ & .5 & $0(0 \%)$ & $1(4 \%)$ & .4 \\
\hline BUN (mg/dL) & $15 \pm 8$ & $10 \pm 6$ & .2 & $11 \pm 5$ & $8 \pm 5$ & .04 \\
\hline Blood creatinine (mg/dL) & $0.5 \pm 0.2$ & $0.6 \pm 0.1$ & .2 & $0.4 \pm 0.1$ & $0.4 \pm 0.1$ & .2 \\
\hline
\end{tabular}

Data presented as mean \pm SD or number (\%). UVH, Univentricular heart; TGA, d-transposition of great arteries; $L R S$, left to right shunt; TOF, tetralogy of Fallot; $H L H S$, hypoplastic left heart syndrome; IAA, interruption of aorta; $H A A$, hypoplastic aortic arch; DORV, double outlet right ventricle; $H R V$, hypoplastic right ventricle; $V S D$, ventricular septal defect; AVSD, atrioventricular septal defect; $H R$, heart rate; $M A P$, mean arterial pressure; $P_{1} E_{1}$, prostaglandin $\mathrm{E}_{1} ; B U N$, blood urea nitrogen. $* P$ values for comparisons between groups I and II (neonates) and between groups III and IV (infants). †Of 24 patients, 22 underwent right ventricle-pulmonary artery conduit, 1 modified Blalock-Taussig shunt, and 1 an aortopulmonary shunt.

\section{RESULTS}

\section{Subjects and Outcomes by Patient Group}

A total of 115 neonates and infants were enrolled in the study, with 24 patients in group I (UVH), 11 in group II (TGA), 55 in group III (LRS), and 25 in group IV (TOF). The patients' preoperative characteristics are listed in Table 1. The neonates in groups I and II ranged in age from 1 to 35 days, and the infants in groups III and IV ranged in age from 1 to 12 months.

The patients' intraoperative and postoperative characteristics are listed in Table 2, with comparisons between the 2 neonatal and 2 infant groups presented. In the neonates, the CPB times were similar; however, the UVH group had shorter crossclamp times than the TGA group ( $47 \pm 12$ vs $59 \pm 12$ minutes, $P<.01$ ). The 12 -hour inotrope scores were similar; however, patients in the UVH group had greater 12-hour serum lactate levels, longer hospital stays, and a longer duration of postoperative mechanical ventilation than the TGA group. The incidence of LCOS and the need for ECLS were greater in the UVH patients, although these differences did not reach statistical significance. No neonates had a major poor outcome in the TGA group compared with 4 patients with a major poor outcome in the UVH group. All these newborns were supported with ECLS and died within the index hospitalization (Table 3).

TABLE 2. Intraoperative and postoperative characteristics

\begin{tabular}{|c|c|c|c|c|c|c|}
\hline & $\begin{array}{c}\text { Group I } \\
(\mathrm{UVH}, \mathbf{n}=\mathbf{2 4})\end{array}$ & $\begin{array}{c}\text { Group II } \\
(\text { TGA, }, \mathbf{n}=11)\end{array}$ & $P$ value* & $\begin{array}{c}\text { Group III } \\
(\text { LRS, } \mathbf{n}=\mathbf{5 5})\end{array}$ & $\begin{array}{c}\text { Group IV } \\
(\mathrm{TOF}, \mathbf{n}=\mathbf{2 5})\end{array}$ & $P$ value* \\
\hline CPB time $(\min )$ & $127 \pm 25$ & $122 \pm 16$ & .31 & $78 \pm 33$ & $105 \pm 43$ & $<.01$ \\
\hline Crossclamp time (min) & $47 \pm 12$ & $59 \pm 12$ & $<.01$ & $52 \pm 20$ & $55 \pm 25$ & .98 \\
\hline 12-h Inotrope score & $13 \pm 6$ & $12 \pm 6$ & .72 & $7 \pm 6$ & $7 \pm 6$ & .88 \\
\hline 12-h Serum lactate $(\mathrm{mmol} / \mathrm{L})$ & $4.5 \pm 2.3$ & $2.8 \pm 1.7$ & .03 & $1.3 \pm 0.8$ & $1.4 \pm 0.7$ & .4 \\
\hline Hospital length of stay (d) & $31(24-42)$ & $17(14-23)$ & $<.01$ & $6(5-9)$ & $8(6-11)$ & .09 \\
\hline Mechanical ventilation duration (d) & $6(3-12)$ & $3(2-5)$ & .03 & $0.8(0.3-1)$ & $1.1(0.7-3)$ & $<.01$ \\
\hline LCOS & $19(79 \%)$ & $6(55 \%)$ & .13 & $10(18 \%)$ & $9(36 \%)$ & .08 \\
\hline ECLS required & $4(17 \%)$ & $0(0 \%)$ & .15 & $0(0 \%)$ & $1(4 \%)$ & .81 \\
\hline Major poor outcomes & $4(17 \%)$ & $0(0 \%)$ & .15 & $2(3 \%)$ & $3(12 \%)$ & .17 \\
\hline
\end{tabular}

Data presented as mean $\pm \mathrm{SD}$, number (\%), or median (interquartile range). UVH, Univentricular heart; TGA, d-transposition of great arteries; $L R S$, left to right shunt; TOF, tetralogy of Fallot; $C P B$, cardiopulmonary bypass; $E C L S$, extracorporeal life support; $L C O S$, low cardiac output status. $* P$ values between groups I and II (neonates) and between groups III and IV (infants). 
TABLE 3. Summary of patients with major poor outcome

\begin{tabular}{cllr}
\hline Group & \multicolumn{1}{c}{ Pt. no., diagnosis, and procedure } & \multicolumn{1}{c}{$\begin{array}{c}\text { Major poor outcome } \\
\text { (within 6 mo) }\end{array}$} & $\begin{array}{r}\text { Postoperative interval } \\
\text { to outcome (d) }\end{array}$ \\
\hline I (UVH) & 1, HLHS after Norwood and RVPA shunt & ECLS, death & 3,6 \\
& 2, HLHS after Norwood and RVPA shunt & ECLS, death & 2,24 \\
& 3, Schone's complex after Norwood & ECLS, death & 15,36 \\
& and RVPA shunt & & \\
& 4, HLHS after Norwood and BTS & ECLS, death & 4,25 \\
III (LRS) & 5, AVSD after 2 patch repair & Death & 41 \\
& 6, AVSD after 2 patch repair & Mitral valve repair & 21 \\
IV (TOF) & 7, TOF after RVPA conduit, VSD closure & ECLS, death & 1,10 \\
& 8, TOF after TAP, VSD closure & VSD patch leak repair & 8 \\
& 9, TOF after TAP, VSD closure & Outflow tract revision & 35 \\
\hline
\end{tabular}

Pt. no., Patient number; $U V H$, univentricular heart; $H L H S$, hypoplastic left heart syndrome; $R V P A$, right ventricle to pulmonary artery; $E C L S$, extracorporeal life support; $B T S$, Blalock-Taussig shunt; $L R S$, left to right shunt; $A V S D$, atrioventricular septal defect; $T O F$, tetralogy of Fallot; TAP, transannular patch; VSD, ventricular septal defect.

In the infant populations, the CPB times were longer in the TOF group than in the LRS group $(105 \pm 43$ vs $78 \pm$ 33 minutes, $P<.01)$. The 12-hour lactate and inotrope scores were similar between the TOF and LRS groups. The incidence of LCOS and hospital length of stay were greater in the TOF group, but these differences did not reach statistical significance. The patients in the TOF group had longer durations of mechanical ventilation $(P<.01)$. There were 3 patients with a major poor outcome in the TOF group, including 1 death and 2 unplanned interventions. The patient who died was also supported by ECLS. In the LRS group, there were 2 major poor outcomes: 1 death and 1 unplanned intervention. All major poor outcomes in the infant groups occurred during the index hospitalization (Table 3).

\section{Perioperative BNP Levels}

The BNP levels preoperatively and at 2, 12, and 24 hours postoperatively for all 4 groups are shown in Figure 1. As

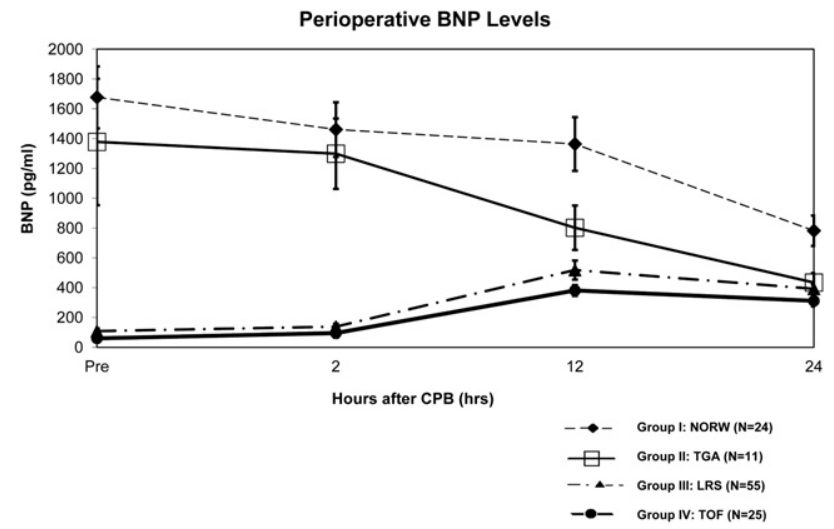

FIGURE 1. Comparison of B-type natriuretic peptide $(B N P)$ before and after cardiopulmonary bypass in all 4 diagnostic groups. NORW, Norwood procedure (univentricular heart); TGA, d-transposition of the great arteries; $L R S$, left to right shunt; TOF, tetralogy of Fallot. Data presented as mean \pm $\mathrm{SD}$. Analysis of variance analysis within all groups, $P<.01$. expected, the preoperative BNP levels were greater in the neonates than in the infants $(1583 \pm 1140$ vs $93 \pm 135$ $\mathrm{pg} / \mathrm{mL}, P<.01$ ). In both neonatal groups (UVH and TGA), the BNP levels were greatest in the preoperative period, decreasing significantly by 24 hours after CPB (repeated measures analysis of variance, $P<.01$ for both groups). In contrast, the BNP levels were lowest in the preoperative period in the infant groups (LRS and TOF). In these groups, the BNP levels peaked at the 12-hour point $(P<.01$ compared with the preoperative levels).

\section{Preoperative BNP Levels and Outcomes}

Within each group, no association was found between the preoperative BNP levels and any of the demographics or postoperative primary or secondary outcomes (data not shown). In the LRS group, the preoperative BNP levels correlated with all postoperative BNP levels, although most strongly with the 2-hour levels ( 2 hours, rho $=0.78$, $P<.001 ; 12$ hours, rho $=0.31, P=.01$; and 24 hours, rho $=0.40, P<.01)$. In the other groups, the preoperative BNP levels correlated with the postoperative BNP levels only at 2 hours $(\mathrm{UVH}$, rho $=0.65, P<.001$; TGA, rho $=0.72, P=.01$; TOF, rho $=0.64, P<.001)$.

\section{Postoperative BNP Levels and Major Poor Outcome}

In the UVH group, the 12- and 24-hour BNP levels were greater in patients with a major poor outcome than in those with a good outcome (12 hours, $1776 \pm 329$ vs $1159 \pm 204 \mathrm{pg} / \mathrm{mL}, P=.04 ; 24$ hours, $1188 \pm 88$ vs $593 \pm 88 \mathrm{pg} / \mathrm{mL}, P<.01$; Figure 2$)$. The perioperative characteristics of the patients in the UVH group stratified by outcome are summarized in Table 4 . Neonates with a poor outcome had greater 12-hour inotrope scores and serum lactate levels, although these differences were not significant.

The 12- and 24-hour BNP levels were greater in patients with a major poor outcome than in those with a good outcome in the LRS group (12 hours, $1215 \pm 375$ vs $492 \pm 62 \mathrm{pg} / \mathrm{mL}$, 
Group I (UVH): BNP Levels

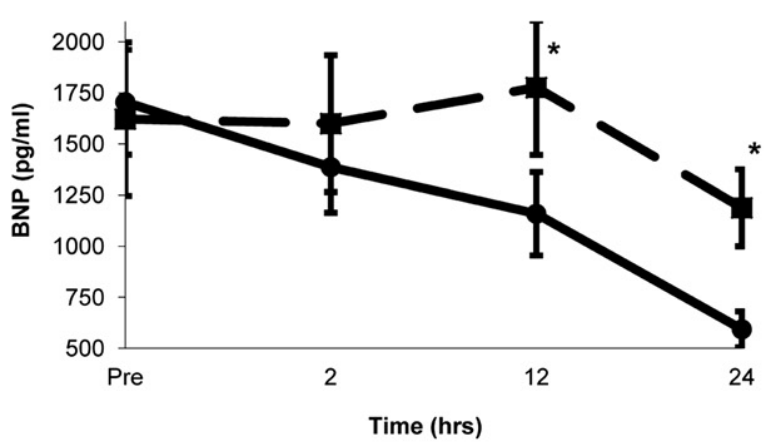

Group I (UVH): Post/Pre BNP Ratio

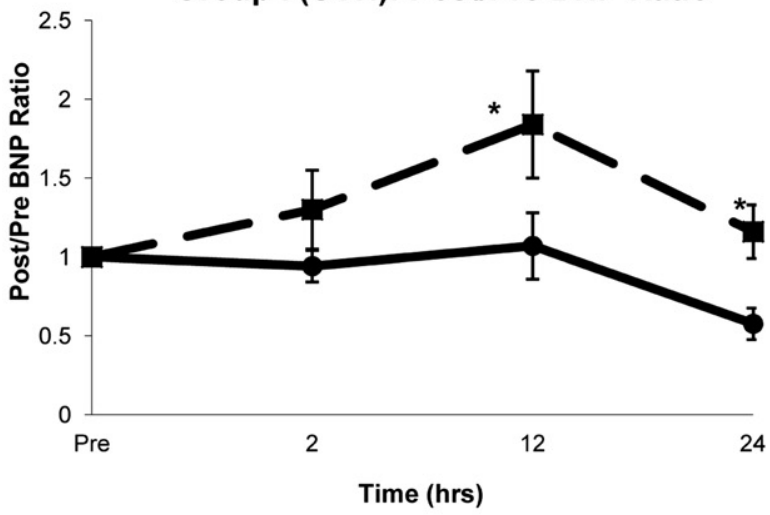

Poor Outcome $(n=4) \longrightarrow$ Good Outcome $(n=20)$

FIGURE 2. Group I (univentricular heart $[U V H]$ ). Comparisons of changes in, Left, absolute B-type natriuretic peptide $(B N P)$ levels and, Right, postoperative/preoperative (post/pre) BNP ratios over time between patients with good outcomes $(\mathrm{n}=20)$ and patients with poor outcomes $(\mathrm{n}=4)$. Both absolute and ratios of BNP were greater at 12 and 24 hours after cardiopulmonary bypass in poor outcome group. Data presented as mean \pm SD. $* P<.05$ vs good outcome (Student $t$ test).

$P=.04 ; 24$ hours, $822 \pm 44$ vs $375 \pm 28 \mathrm{pg} / \mathrm{mL}, P=.02)$ and TOF group (12 hours, $476 \pm 53$ vs $368 \pm 40 \mathrm{pg} / \mathrm{mL}, P=.04$; 24 hours, $378 \pm 92$ vs $302 \pm 41 \mathrm{pg} / \mathrm{mL}, P=.04)$. Figures 3 and 4 demonstrate the BNP levels stratified by outcome for the infant groups. The characteristics of the patients in the LRS and TOF groups stratified by outcome are summarized in Table 5.

TABLE 4. Characteristics of group I (UVH) patients stratified by primary outcome

\begin{tabular}{lccc}
\hline & \multicolumn{2}{c}{ Group I (UVH, n= 24) } \\
\cline { 2 - 4 } & $\begin{array}{c}\text { Good } \\
\text { outcome }\end{array}$ & $\begin{array}{c}\text { Poor } \\
\text { outcome }\end{array}$ & P value \\
\hline Patient number (\%) & $20(83 \%)$ & $4(17 \%)$ & \\
Age (d) & $10 \pm 8$ & $6 \pm 3$ & .4 \\
Weight (kg) & $3.4 \pm 0.5$ & $3.2 \pm 0.4$ & .5 \\
CPB time (min) & $128 \pm 26$ & $118 \pm 21$ & .5 \\
Crossclamp time (min) & $47 \pm 13$ & $46 \pm 9$ & .9 \\
12-h Post-CPB characteristics & & & \\
HR (min ${ }^{-1}$ ) & $165 \pm 12$ & $159 \pm 15$ & .4 \\
MAP (mm Hg) & $54 \pm 9$ & $50 \pm 7$ & .4 \\
Inotrope score & $11.5 \pm 5.2 *$ & $21 \pm 8 *$ & .06 \\
Serum lactate (mmol/L) & $4.1 \pm 2.1$ & $6.5 \pm 2.3$ & .06 \\
BUN (mg/dL) & $16 \pm 7$ & $17 \pm 8$ & .9 \\
Blood creatinine (mg/dL) & $0.68 \pm 0.2$ & $0.9 \pm 0.4$ & .2 \\
Secondary outcome characteristics & & & \\
$\quad$ Hospital length of stay (d) & $31(24-39)$ & $43(22-145)$ & .5 \\
$\quad$ Mechanical ventilation & $6(3-12)$ & $13(6-52)$ & .4 \\
$\quad$ duration (d) & & & \\
LCOS & $15(75 \%)$ & $4(100 \%)$ & .54 \\
\hline
\end{tabular}

Data presented as mean $\pm \mathrm{SD}$, number (\%), or median (interquartile range). No patients in group II (d-transposition of great arteries) had 6-month poor outcomes. $U V H$, Univentricular heart; $C P B$, cardiopulmonary bypass; $H R$, heart rate; $M A P$, mean arterial pressure; $B U N$, blood urea nitrogen; $L C O S$, low cardiac output syndrome. $*^{*}$ Good outcome, $\mathrm{n}=11$; poor outcome, $\mathrm{n}=2$.

\section{Perioperative BNP Change and Primary and Secondary Outcomes}

Because absolute BNP levels appear to be age and lesion specific, and preoperative BNP levels are variable, we also examined the relationship between the change in BNP during the first 24 hours after surgery and both primary and secondary outcomes. To accomplish this, we standardized the patients' postoperative BNP levels to their preoperative levels, expressed as a postoperative/preoperative (post/ pre) BNP ratio. The right panels of Figures 2 through 4 show the post/pre BNP ratios at each point for the patients with good and poor postoperative outcomes for the UVH, LRS, and TOF groups, respectively.

In the UVH group, the post/pre BNP ratios at 12 and 24 hours were greater in patients with a major poor outcome than in those with a good outcome (12 hours, $1.84 \pm 0.34$ vs $1.07 \pm 0.21, P=.04 ; 24$ hours, $1.16 \pm 0.17$ vs $0.58 \pm$ $0.10, P=.03)$. Receiver operating characteristic curves indicated that a 12-hour post/pre BNP ratio greater than 1.0 was $63 \%$ sensitive and $81 \%$ specific for a poor postoperative outcome (area under the curve 0.72 ). In the UVH group, the post/pre BNP ratios at 12 and 24 hours were greater in patients with a mechanical ventilation duration of 7 days or longer $(\mathrm{n}=10)$ compared with those with less than 7 days (12-hour ratio, $2.28 \pm 0.3$ vs $0.65 \pm 0.8, P=.03$, 24-hour ratio, $1.32 \pm 0.4$ vs $0.38 \pm 0.5, P=.03$ ). The post/pre BNP ratios at 12 hours were also greater in patients with a hospital length of stay of 30 days or longer $(n=12)$ than in those with a length of stay shorter than 30 days (12hour ratio, $2.0 \pm 0.4$ vs $0.65 \pm 0.2, P=.02$ ). The absolute BNP levels were not different between patients with these secondary outcomes at any point, indicating that the change in BNP levels was a better predictor of these outcomes than the absolute levels. No differences were found in the BNP 


\section{Group III (LRS): BNP Levels}

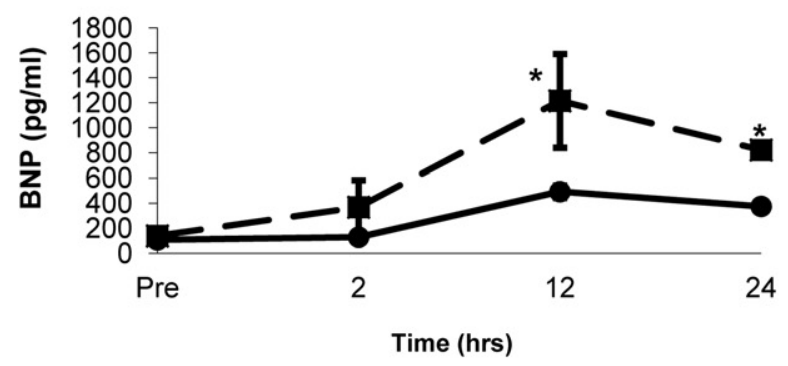

Group III (LRS): Post/Pre BNP Ratio

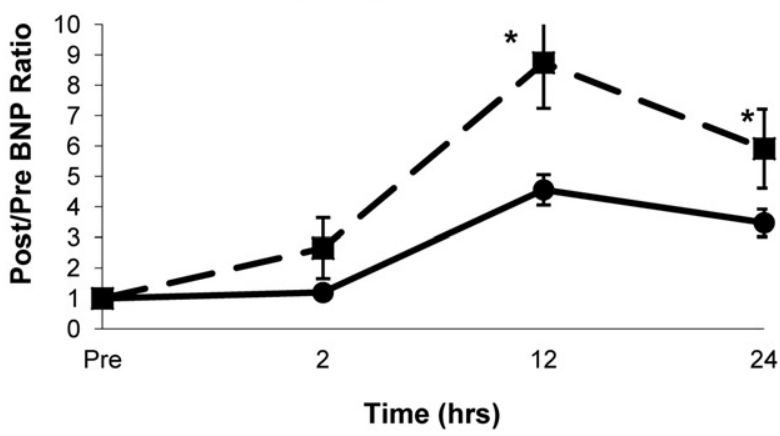

FIGURE 3. Group III (left to right shunt $[L R S]$ ). Comparisons of changes in, Left, absolute B-type natriuretic peptide $(B N P)$ levels and, Right, postoperative/preoperative (post/pre) BNP ratios over time between patients with good outcomes $(\mathrm{n}=53)$ and patients with poor outcomes $(\mathrm{n}=2)$. Both absolute and ratios of BNP were greater at 12 and 24 hours after cardiopulmonary bypass in poor outcome group. Data presented as mean $\pm \mathrm{SD}$. $* P<.05$ vs good outcome (Student $t$ test).

ratios between patients with and without these secondary outcomes in the TGA group $(\mathrm{n}=2$ and $\mathrm{n}=1$ for prolonged ventilation and hospitalization, respectively).

In the LRS and TOF groups, the 12- and 24-hour post/pre BNP ratios were greater in patients with poor outcomes than in those with good outcomes (Figures 3 and 4). In the TOF group, all 3 patients with a major poor outcome had a 12hour post/pre BNP ratio greater than 45, and all patients with a good outcome had a 12-hour post/pre BNP ratio less than 10 (mean 12-hour post/pre BNP ratio, 29). In the infant populations, no associations were seen between the BNP ratios and our secondary outcomes.

\section{Serum Lactate Levels}

We examined the relationship between the serum lactate level at each sampling point and outcomes for each group, given the common use of lactate levels to detect LCOS in these patients. No significant association was seen between the serum lactate levels and the occurrence of major poor outcomes or our secondary outcomes of prolonged mechanical ventilation and prolonged hospitalization. In addition, the postoperative/preoperative serum lactate ratios were not associated with our primary or secondary outcomes (data not shown).

\section{Other Variables}

Physiologic parameters such as heart rate, mean arterial pressure, central venous pressure, cerebral oximetry, and pulse oximetry were not associated with our primary or secondary outcomes. In all groups, the changes in these parameters over time between those with a major poor outcome versus a good outcome were also not significant using
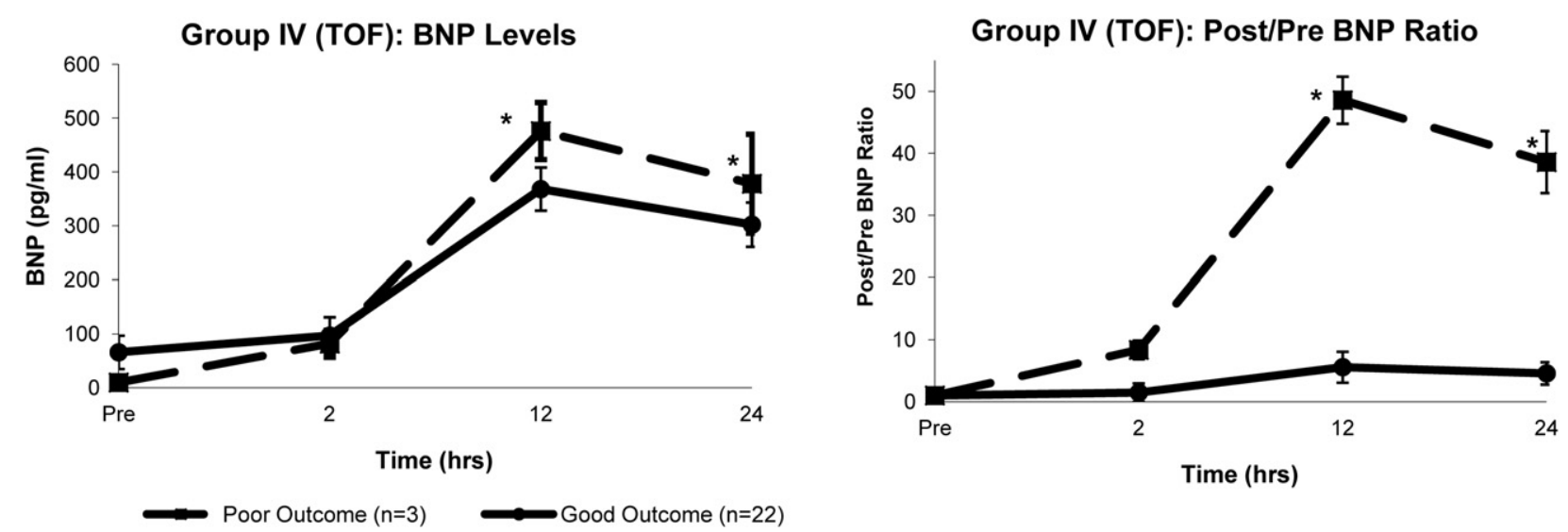

FIGURE 4. Group IV (tetralogy of Fallot [TOF]). Comparisons of changes in, left, absolute B-type natriuretic peptide (BNP) levels and, right, postoperative/preoperative (post/pre) BNP ratios over time between patients with good outcomes $(\mathrm{n}=22)$ and patients with poor outcomes $(\mathrm{n}=3)$. Both absolute values and ratios of BNP were greater at 12 and 24 hours after cardiopulmonary bypass in poor outcome group. Data presented as mean \pm SD. $* P<.05$ vs good outcome (Student $t$ test). 
TABLE 5. Characteristics of groups III and IV stratified by primary outcome

\begin{tabular}{|c|c|c|c|c|c|c|}
\hline & \multicolumn{3}{|c|}{ Group III (LRS; $\mathbf{n}=\mathbf{5 5})$} & \multicolumn{3}{|c|}{ Group IV (TOF; $\mathbf{n}=25)$} \\
\hline & Good outcomes & Poor outcomes & $P$ value & Good outcomes & Poor outcomes & $P$ value \\
\hline Patients & $53(96 \%)$ & $2(4 \%)$ & & $22(88 \%)$ & $3(12 \%)$ & \\
\hline Age (mo) & $10.2 \pm 2.9$ & $4.8 \pm 3.7$ & .3 & $4.6 \pm 2.5$ & $2.2 \pm 1.8$ & .04 \\
\hline Weight $(\mathrm{kg})$ & $6.7 \pm 3.5$ & $4.3 \pm 1.0$ & .1 & $5.7 \pm 1.2$ & $3.8 \pm 1.6$ & $<.01$ \\
\hline CPB time (min) & $73 \pm 29$ & $118 \pm 37$ & $<.01$ & $100 \pm 37$ & $120 \pm 57$ & .3 \\
\hline Crossclamp time (min) & $50 \pm 19$ & $70 \pm 23$ & .02 & $59 \pm 25$ & $40 \pm 24$ & .1 \\
\hline \multicolumn{7}{|l|}{ 12-h Post-CPB characteristics } \\
\hline $\mathrm{HR}\left(\min ^{-1}\right)$ & $125 \pm 23$ & $138 \pm 19$ & .2 & $136 \pm 19$ & $138 \pm 15$ & .8 \\
\hline MAP (mm Hg) & $66 \pm 8$ & $52 \pm 8$ & $<.01$ & $61 \pm 10$ & $62 \pm 14$ & .8 \\
\hline Inotrope score & $6.5 \pm 5.6$ & $8.8 \pm 7.6$ & .4 & $6.5 \pm 4.9$ & $8.7 \pm 9.1$ & .4 \\
\hline Serum lactate $(\mathrm{mmol} / \mathrm{L})$ & $1.2 \pm 0.7$ & $1.6 \pm 1.3$ & .4 & $1.4 \pm 0.7$ & $1.5 \pm 0.5$ & .8 \\
\hline BUN $(\mathrm{mg} / \mathrm{dL})$ & $12.9 \pm 5.9$ & $15.2 \pm 6.0$ & .4 & $11.5 \pm 4.9$ & $13.3 \pm 4.2$ & .5 \\
\hline Blood creatinine $(\mathrm{mg} / \mathrm{dL})$ & $0.44 \pm 0.1$ & $0.56 \pm 0.21$ & .04 & $0.47 \pm 0.14$ & $0.57 \pm 0.15$ & .3 \\
\hline \multicolumn{7}{|l|}{ Secondary outcome characteristics } \\
\hline Hospital length of stay (d) & $6(5-8)$ & $15(10-21)$ & $<.01$ & $8(6-10)$ & $11(5-36)$ & .5 \\
\hline Mechanical ventilation duration (d) & $0.8(0.5-1.0)$ & $5.0(0.5-6.0)$ & .3 & $1.0(0.7-2.9)$ & $1.3(0.7-36)$ & .6 \\
\hline LCOS & $9(19 \%)$ & $1(50 \%)$ & .06 & $7(28 \%)$ & $2(66 \%)$ & .07 \\
\hline
\end{tabular}

Data presented as mean $\pm \mathrm{SD}$, number (\%), or median (interquartile range). $C P B$, Cardiopulmonary bypass; $H R$, heart rate; $M A P$, mean arterial pressure; $B U N$, blood urea nitrogen; $L C O S$, low cardiac output syndrome; $L R S$, left to right shunt; TOF, tetralogy of Fallot.

analysis of variance (data not shown). When individually controlled for other variables, such as renal function, patient age, CPB time, weight, and other demographic and physiologic parameters collected, the associations between BNP and the primary and secondary outcomes remained independent on multivariate analysis. This suggests that BNP is a simple, single predictor that might be a useful adjunct to more complex risk assessments that include factors such as young age, low weight, and CPB time, for example.

\section{DISCUSSION}

These data suggest that BNP is a valuable biomarker for prognostication and risk stratification in neonates and infants undergoing repair or palliation of cardiac defects. However, the present findings indicate that the interpretation of perioperative BNP levels with respect to outcomes will differ according to patient age and perioperative physiologic features (eg, type of defect and surgical procedure). Neonates undergoing a modified Norwood procedure or arterial switch procedure had high preoperative BNP levels that decreased during the first 24 hours after CPB. In contrast, infants undergoing primary repair of a VSD, atrioventricular septal defect, or TOF had lower preoperative BNP levels that increased after $\mathrm{CPB}$, reaching a peak at 12 hours. Furthermore, deviations from the aggregate pattern of change were associated with a 6-month composite poor outcome. Specifically, in neonates undergoing a modified Norwood procedure, a failure of BNP levels to decrease postoperatively was associated with a poor outcome; however, in infants, an exaggerated postoperative increase in BNP was associated with a poor outcome. Although our study did not determine possible etiologies for the differences in the perioperative BNP changes, it highlights the utility of understanding such age- and lesion-dependent differences.

In contrast, absolute BNP levels were not associated with outcomes except in the UVH group. Therefore, our results are consistent with an expanding experience indicating that a single cutoff value is unlikely to provide value across the varied spectrum of pediatric patients undergoing surgery for congenital heart disease. However, these data do suggest that perioperative patterns of change in BNP levels can be established for groups of patients classified by age and perioperative physiologic features. Moreover, deviations from these patterns of change provide valuable clinical insights that are not reproduced by currently available biomarkers or known risk factors (eg, $\mathrm{CPB}$ time, age). The precise reason the BNP levels were independently associated with our primary and secondary outcomes in these patients even after multivariate analysis is unclear. Some theories include volume load on the ventricle not predicted by central venous pressure values, ventricular wall stress not suspected during adequate oxygen delivery (ie, normal lactates or $\mathrm{AVdO}_{2}$ values), or residual lesions not routinely noted in the intensive care unit until a later echocardiogram or catheterization. Additional studies are required to better determine the mechanisms of BNP regulation, including its expression and clearance, to answer these important questions.

In agreement with previous studies, we found wide variability in BNP levels (Figure 1). These previous studies suggested that this variability is influenced in part by age, the type of cardiac defect, and exposure to CPB. ${ }^{12-16}$ Our study of neonatal patients has demonstrated that even agecontrolled patients have significant preoperative variability in their BNP levels. This could explain the poor association 
between preoperative BNP levels with outcomes and highlights the utility of post/pre BNP ratios. It also demonstrates that BNP levels are not dictated by age alone but, rather, by a complex interplay among a patient's age, physiologic features, and anatomy.

Only limited data are available relating perioperative BNP levels to outcomes in any pediatric age group undergoing cardiac surgery for congenital heart disease. An initial study of 51 infants and children with biventricular cardiac defects found that BNP levels increased after surgery, peaking at 12 hours, and that the 12-hour level was associated independently with the need for mechanical ventilation beyond 48 hours and the development of LCOS. ${ }^{17}$ Other studies have examined $\mathrm{N}$ terminal-proBNP levels in infants and children undergoing cardiac surgery. ${ }^{18-20}$ One study demonstrated a peak elevation at 24 hours postoperatively that correlated with postoperative outcomes, including the duration of mechanical ventilation, $\mathrm{CPB}$ length, and inotropic score. ${ }^{20}$ Virtually all the studies of a pediatric cardiac surgical population have found that BNP levels increased postoperatively in infants, consistent with the findings in the infants in the present study. ${ }^{14,17,21,22}$ We also found that these elevations are predictive of postoperative outcomes.

In contrast to this established pattern in infants, the neonates with good outcomes in our study demonstrated a decrease in BNP levels after surgery. No neonate whose BNP level decreased postoperatively relative to their preoperative baseline had a poor postoperative outcome. In contrast, an increase in BNP greater than baseline after surgery was associated with LCOS, a longer duration of mechanical ventilation, and a 6-month composite of poor outcome. A previous study from our institution demonstrated similar findings in 31 neonates with a variety of cardiac defects undergoing repair. ${ }^{23}$ In contrast to these studies, Niedner and colleagues $^{24}$ studied 102 neonates and non-neonatal controls and found that the BNP levels increased after surgery, peaking at 12 hours. The reasons for the differences between that study and our previous and present investigations might relate in part to differences in the lesions of the cohorts, including single and biventricular physiologic types. This highlights the importance of stratification of BNP changes by the specific lesion, instead of combining cohorts, given the variability seen within lesion types.

In the present study, we compared 2 different populations of neonates, those with single ventricle physiologic features and those with TGA; both groups demonstrated a decrease in postoperative BNP levels. Cannesson and colleagues ${ }^{25}$ measured perioperative BNP levels in 30 neonates undergoing an arterial switch operation for TGA. In contrast to our findings in the present, and a previous, study ${ }^{23}$ the BNP levels increased during the first 48 hours postoperatively in their patient population. However, similar to our studies, these levels were predictive of important postoperative outcomes. The reasons for the differences in the pattern of postoperative BNP changes between these studies are unclear, particularly because the populations were similar with respect to age and cardiac defect. These data suggest that although particular patterns of BNP change within an age and lesion might be predictive of postoperative outcomes, the specific patterns that are predictive might be institution specific. Additional studies are warranted to address this important disparity.

To date, the 2 most commonly used biomarkers to monitor patients after congenital cardiac surgery are the $\mathrm{AVdO}_{2}$ and serum lactate. ${ }^{1,26-28}$ The $\mathrm{AVdO}_{2}$ is a relatively reliable indicator of the balance between oxygen delivery and oxygen consumption and, thus, has been used successfully to guide postoperative therapies. ${ }^{26}$ Several studies of serum lactate have found that lactate, and its rate of change in the postoperative period, predicted the outcome, including mortality. ${ }^{1,27}$ However, these findings have not been reproduced by other larger studies, and the precise lactate levels that were predictive varied considerably, obviating a standardized use of lactate as a prognostic biomarker. ${ }^{1,27,28}$ However, because acute management decisions are aimed at normalizing the venous oxyhemoglobin saturation and lactate levels, it is likely that only persistently abnormal values refractory to treatment will be predictive of an adverse event in the short term. Therefore, it was not surprising that serum lactate levels and postoperative changes in serum lactate failed to predict postoperative outcomes in the present study. However, the data were insufficient to analyze the utility of $\mathrm{AVdO}_{2}$ in our study. Thus, in our study, the postoperative change in BNP was unique in its ability to predict outcomes. However, these results might reflect that clinicians were unaware of the BNP levels and thus were unable to knowingly modify the factors contributing to the elevation in BNP.

Although the present study is the largest study of perioperative BNP levels in neonates and infants undergoing surgery for congenital cardiac defects reported to date, its primary limitation related to the limited number of patients with outcomes of interest. For example, the TGA group had no poor outcomes, and the other 3 groups had 2 to 4 patients with poor outcomes. In addition, several important congenital heart defects were not included in our study. Thus, larger studies are needed to precisely define the role of BNP in the management of congenital heart disease in neonates and infants.

These data suggest that additional age- and lesionspecific characterizations of perioperative BNP levels in neonatal and pediatric patients with congenital heart disease could lead to the establishment of BNP as an important biomarker to predict both short- and long-term clinical outcomes.

The authors would like to thank the Pediatric Critical Care Fellows, the Cardiac Intensive Care Nurses, the Pediatric Clinical 
Research Center, Swati Antala, and Yarden Fraiman for their invaluable assistance with our study.

\section{References}

1. Charpie JR, Dekeon MK, Goldberg CS, Mosca RS, Bove EL, Kulik TJ. Serial blood lactate measurements predict early outcome after neonatal repair or palliation for complex congenital heart disease. J Thorac Cardiovasc Surg. 2000;120: 73-80.

2. Jenkins KJ, Gauvreau K, Newburger JW, Spray TL, Moller JH, Iezzoni LI. Consensus-based method for risk adjustment for surgery for congenital heart disease. J Thorac Cardiovasc Surg. 2002;123:110-8

3. Doust JA, Glasziou PP, Pietrzak E, Dobson AJ. A systematic review of the diagnostic accuracy of natriuretic peptides for heart failure. Arch Intern Med. 2004; 164:1978-84

4. Doust JA, Pietrzak E, Dobson A, Glasziou P. How well does B-type natriuretic peptide predict death and cardiac events in patients with heart failure: systematic review. BMJ. 2005;330:625.

5. Troughton RW, Frampton CM, Yandle TG, Espiner EA, Nicholls MG, Richards AM. Treatment of heart failure guided by plasma aminoterminal brain natriuretic peptide (N-BNP) concentrations. Lancet. 2000;355:1126-30.

6. Costello JM, Goodman DM, Green TP. A review of the natriuretic hormone system's diagnostic and therapeutic potential in critically ill children. Pediatr Crit Care Med. 2006; 7:308-18.

7. Price JF, Thomas AK, Grenier M, Eidem BW, O'Brian Smith E, Denfield SW, et al. B-type natriuretic peptide predicts adverse cardiovascular events in pediatric outpatients with chronic left ventricular systolic dysfunction. Circulation. 2006; 114:1063-9.

8. Checchia PA. Perioperative mechanical circulatory support in children with critical heart disease. Curr Treat Options Cardiovasc Med. 2011;13:414-24.

9. Domico MB, Ridout DA, Bronicki R, Anas NG, Cleary JP, Cappon J, et al. The impact of mechanical ventilation time before initiation of extracorporeal life support on survival in pediatric respiratory failure: a review of the extracorporeal life support registry. Pediatr Crit Care Med. 2011;13:16-21

10. Hoffman TM, Wernovsky G, Atz AM, Kulik TJ, Nelson DP, Chang AC, et al. Efficacy and safety of milrinone in preventing low cardiac output syndrome in infants and children after corrective surgery for congenital heart disease. Circulation. 2003;107:996-1002.

11. Wernovsky G, Wypij D, Jonas RA, Mayer JE Jr, Hanley FL, Hickey PR, et al. Postoperative course and hemodynamic profile after the arterial switch operation in neonates and infants: a comparison of low-flow cardiopulmonary bypass and circulatory arrest. Circulation. 1995;92:2226-35.

12. Koch A, Singer H. Normal values of B type natriuretic peptide in infants, children, and adolescents. Heart. 2003;89:875-8.

13. Suda K, Matsumura M, Matsumoto M. Clinical implication of plasma natriuretic peptides in children with ventricular septal defect. Pediatr Int. 2003;45:249-54.

14. Costello JM, Backer CL, Checchia PA, Mavroudis C, Seipelt RG, Goodman DM. Alterations in the natriuretic hormone system related to cardiopulmonary bypass in infants with congestive heart failure. Pediatr Cardiol. 2004;25:347-53.
15. Law YM, Keller BB, Feingold BM, Boyle GJ. Usefulness of plasma B-type natriuretic peptide to identify ventricular dysfunction in pediatric and adult patients with congenital heart disease. Am J Cardiol. 2005;95:474-8.

16. Law YM, Ettedgui J, Beerman L, Maisel A, Tofovic S. Comparison of plasma Btype natriuretic peptide levels in single ventricle patients with systemic ventricle heart failure versus isolated cavopulmonary failure. Am J Cardiol. 2006;98: 520-4.

17. Shih CY, Sapru A, Oishi P, Azakie A, Karl TR, Harmon C, et al. Alterations in plasma B-type natriuretic peptide levels after repair of congenital heart defects: a potential perioperative marker. J Thorac Cardiovasc Surg. 2006;131 632-8.

18. Mir TS, Haun C, Lilje C, Laer S, Weil J. Utility of N-terminal brain natriuretic peptide plasma concentrations in comparison to lactate and troponin in children with congenital heart disease following open-heart surgery. Pediatr Cardiol. 2006;27:209-16.

19. Gessler P, Knirsch W, Schmitt B, Rousson V, von Eckardstein A. Prognostic value of plasma $\mathrm{N}$-terminal pro-brain natriuretic peptide in children with congenital heart defects and open-heart surgery. J Pediatr. 2006; 148:372-6.

20. Perez-Piaya M, Abarca E, Soler V, Coca A, Cruz M, Villagra F, et al. Levels of $\mathrm{N}$-terminal-pro-brain natriuretic peptide in congenital heart disease surgery and its value as a predictive biomarker. Interact Cardiovasc Thorac Surg. 2011;12: 461-6.

21. Koch A, Kitzsteiner T, Zink S, Cesnjevar R, Singer H. Impact of cardiac surgery on plasma levels of B-type natriuretic peptide in children with congenital heart disease. Int J Cardiol. 2007;114:339-44

22. Watanabe M, Egi K, Hasegawa S, Tanaka H, Ohshima H, Sakamoto T, et al. Significance of serum atrial and brain natriuretic peptide release after coronary artery bypass grafting. Surg Today. 2003;33:671-3.

23. Hsu JH, Keller RL, Chikovani O, Cheng H, Hollander SA, Karl TR, et al. B-type natriuretic peptide levels predict outcome after neonatal cardiac surgery J Thorac Cardiovasc Surg. 2007;134:939-45.

24. Niedner MF, Foley JL, Riffenburgh RH, Bichell DP, Peterson BM, Rodarte A B-type natriuretic peptide: perioperative patterns in congenital heart disease. Congenit Heart Dis. 2010;5:243-55.

25. Cannesson M, Bionda C, Gostoli B, Raisky O, di Filippo S, Bompard D, et al Time course and prognostic value of plasma B-type natriuretic peptide concentration in neonates undergoing the arterial switch operation. Anesth Analg. 2007; 104:1059-65.

26. Tweddell JS, Hoffman GM. Postoperative management in patients with complex congenital heart disease. Semin Thorac Cardiovasc Surg Pediatr Card Surg Апnu. 2002;5:187-205.

27. Cheung PY, Etches PC, Weardon M, Reynolds A, Finer NN, Robertson CM. Use of plasma lactate to predict early mortality and adverse outcome after neonatal extracorporeal membrane oxygenation: a prospective cohort in early childhood Crit Care Med. 2002;30:2135-9.

28. De Oliveira NC, Ashburn DA, Khalid F, Burkhart HM, Adatia IT, Holtby HM et al. Prevention of early sudden circulatory collapse after the Norwood operation. Circulation. 2004;110(11 Suppl. 1):II133-8. 